\title{
OTEC Thermal Resource Report for Manila, Philippine Islands
}

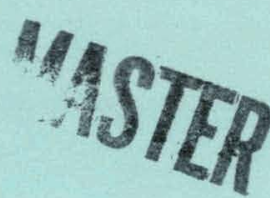

May 1979

Prepared for

U.S. Department of Energy

Assistant Secretary for Energy Technology

Division of Central Solar Technology

Under Contract No. ET-78-C-01-2898 


\section{DISCLAIMER}

This report was prepared as an account of work sponsored by an agency of the United States Government. Neither the United States Government nor any agency Thereof, nor any of their employees, makes any warranty, express or implied, or assumes any legal liability or responsibility for the accuracy, completeness, or usefulness of any information, apparatus, product, or process disclosed, or represents that its use would not infringe privately owned rights. Reference herein to any specific commercial product, process, or service by trade name, trademark, manufacturer, or otherwise does not necessarily constitute or imply its endorsement, recommendation, or favoring by the United States Government or any agency thereof. The views and opinions of authors expressed herein do not necessarily state or reflect those of the United States Government or any agency thereof. 


\section{DISCLAIMER}

Portions of this document may be illegible in electronic image products. Images are produced from the best available original document. 


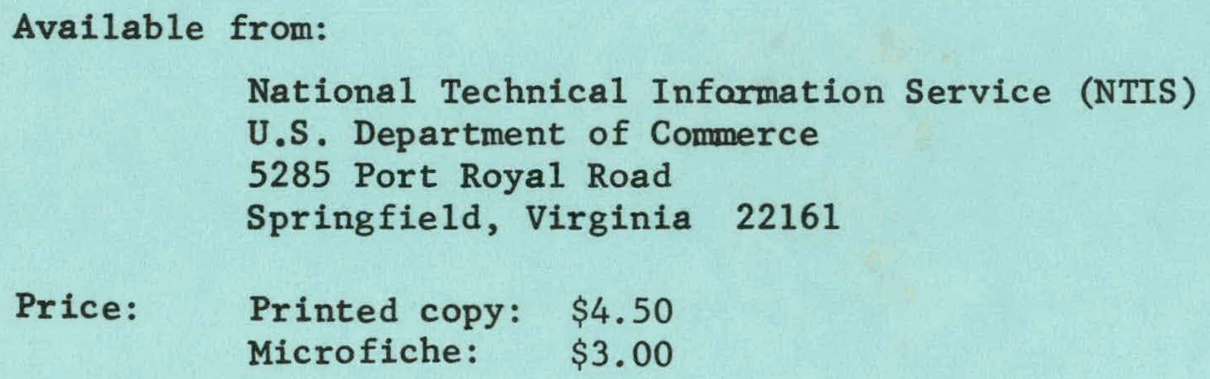




\section{NOTICE}

This roport was propared as an oooount af worle sponsored by the United States Government. Neither the United States nor the United States Department of Energy, nor any of their employees, makes any warranty, express or implied, or assumes any legal liability or responsibility for the accuracy; completeness, or usefulness of any information, apparatus, product, or process disclosed, or represents that its use wowld nut infringe privately owned fights. Reference . herein to any specific commercial product, process, or service by trade name, mork, manufacturer, or otherwise, does not necessarily constitute or imply its endorsement, recommendation, or favoring by the United States Government or any ageney thereof. The views and opinions of au thors expressed herein do not necessarily state or reflect those of the United States Government or any agency thereof. . 
INTRODUCTION $\ldots \ldots \ldots \ldots \ldots \ldots \ldots \ldots \ldots \ldots \ldots \ldots \ldots \ldots \ldots \ldots \ldots$

I. EXECUTIVE SUMMARY................ I-I

II. BATHYMETRY..................... II-I

III. THERMAL RESOURCE. III-1

IV. MIXED LAYER DEPTH................ IV-1

v. WEATHER CONDITIONS................ V-I

VI. SEA AND SWELL CHARACTERISTICS. VI-1

VII. CURRENTS. VII -1

REFERENCES. $\mathrm{R}-1$ 
One of the basic environmental considerations in site selection for an Ocean Thermal Energy Conversion (OTEC) power plant is the availability of an adequate temperature difference resource: OTEC plants are designed to convert the potential energy in the temperature difference between the warm ocean surface water and the cold water existing at deeper depths into electricity. The turbines which produce the electricity must run on temperature differentials which are extremely small by the standards of conventional energy plants. Therefore, a definition of the most probable temperature structure for a site is most important.

In order to define temperäture structures for OTEC areas of interest, Ocean Data sýstems, Inc. (ODSI) has developed computer data filès of all unclásified soundings available. The primary sources for the data were NOAA's National Oceanographic Data Center, 'and the U.S. Navy's Fleet Numerical Weather Central: The files were updated in September 1978. Inciuded in the data base were mechanical bathythermographs (MBT), expendabie bathythermographs (XBT), salinity temperature depth systems (STD), and Nansen casts.

Under U.S. Departmënt of Energy, Division of Solar Energy Contract No. EY-78-C-2989, ODSI has analyzed the monthly temperature structure for ten different geographical areas. The ten sites selected for study by the Department of Energy are: 


\section{Latitude Longitude}

- Bottom Mounted or

Shore Plants: Dampier Land $13-18^{\circ} \mathrm{S} \quad 118-121^{\circ} \mathrm{E}$

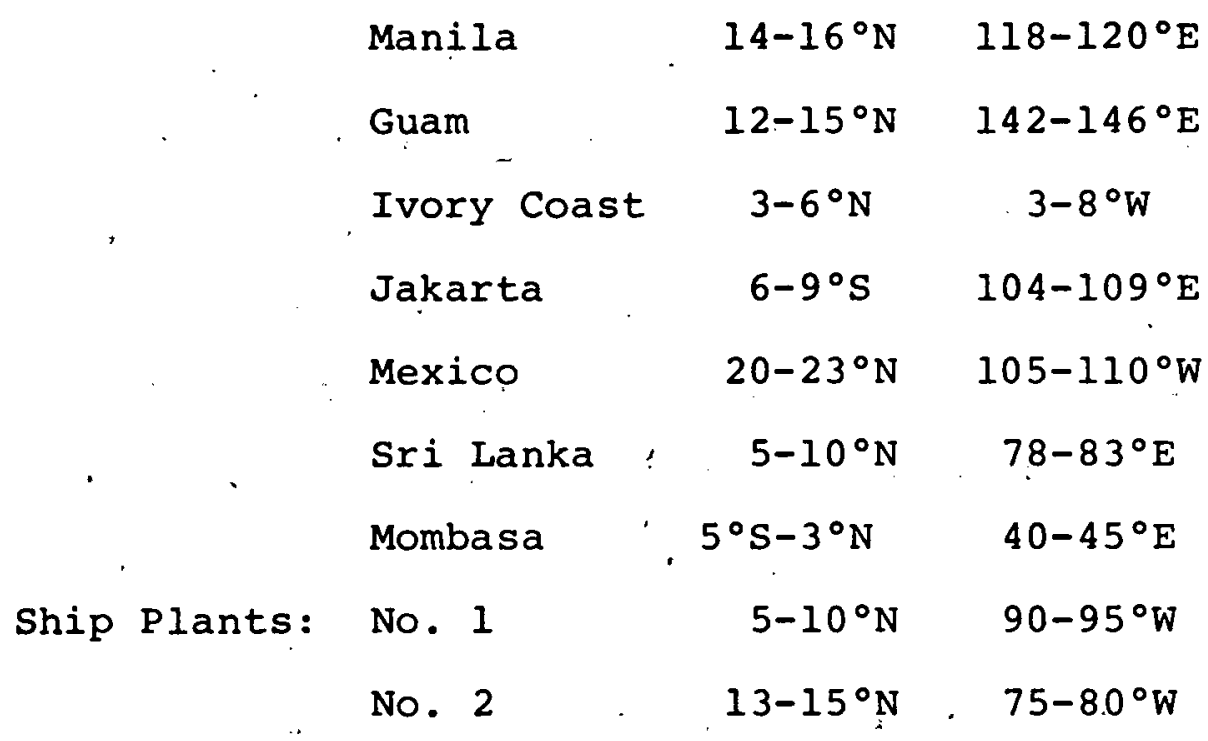

The location of these sites is shown on the following map; a separate report was produced for each site.

For each area, the most probable temperature structures were determined. When the most probable temperature soundings were plotted, some month to month variability was present which was caused by the non-uniform data sample rather than by real changes in the ocean. These short-period time variations were removed by a filtering process described by Wolff, et al (1977), [44]. Availability of cold and warm water was examined at each site. In addition to warm and cold water availability, there are other requirements for the continuous operation of an OTEC plant. An adequate temperature differential $(\Delta T)$ is the primary need. A $\Delta T$ greater than $16.7^{\circ} \mathrm{C}\left(30^{\circ} \mathrm{F}\right)$ for the coldest month of the year would enable year round operation. The annual mean $\Delta T$ for 


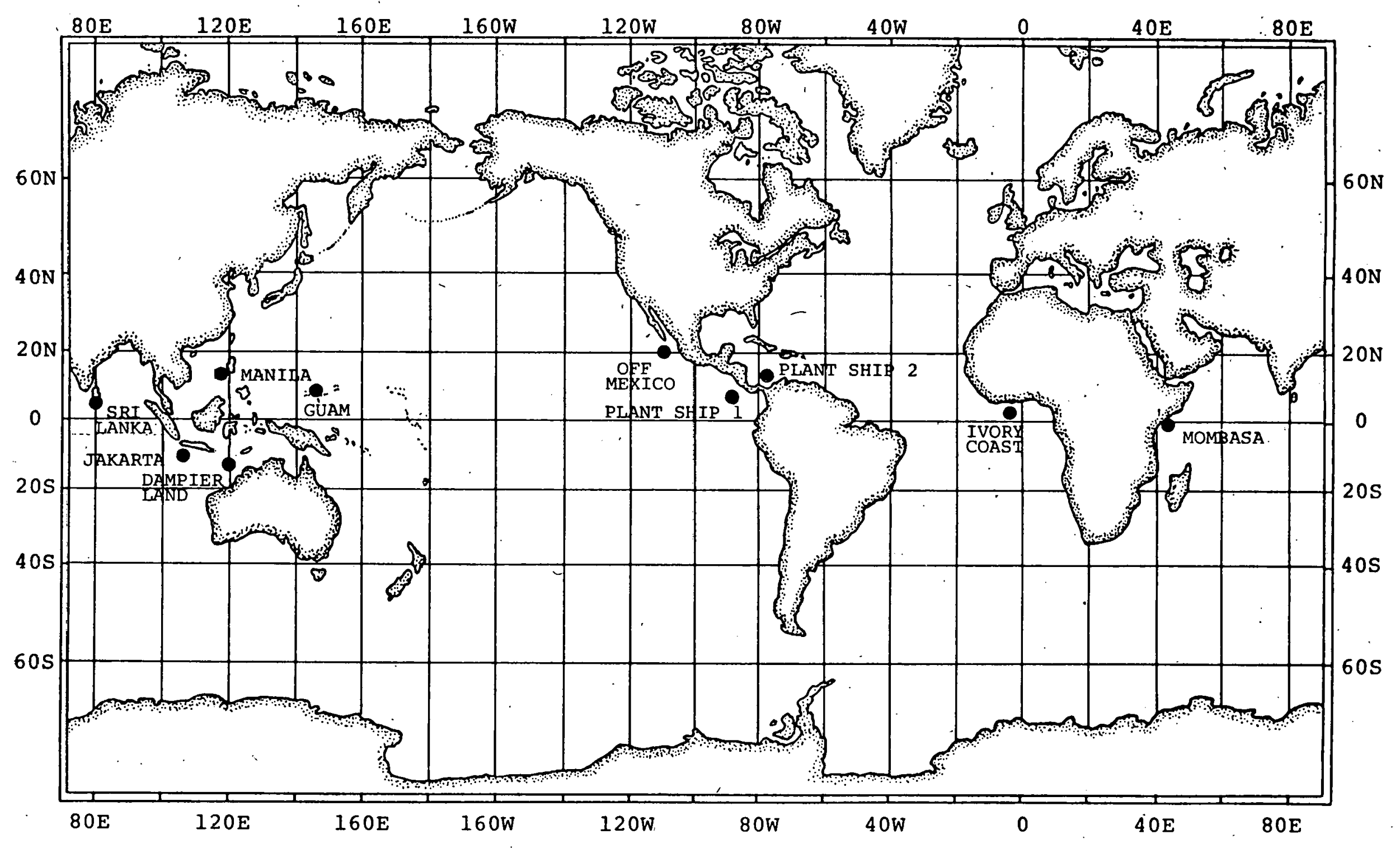

LOCATION OF THE TEN SELECTED SITES 
a site should equal or exceed $20^{\circ} \mathrm{C}\left(36^{\circ} \mathrm{F}\right)$. Besides the thermal resource, there are other operational requirements. Bottom depth should be less than 1500 meters for mooring. Minimum distances offshore to 1000-meter depth is another important consideration for shore plants. Currents should be sufficient to guarantee good cold/warm water sources and to provide for dispersion of modified water. Desirable sites also have light winds, minimum sea and swell, and the. lack of severe storms. These parameters have been examined for each site. The following tables summarize some of the key site parameters for each location. 
SUMMARY OF SITE PARAMETERS

\begin{tabular}{|c|c|c|c|c|c|c|}
\hline Site & \begin{tabular}{|c|} 
Monthly Mean \\
Temperature $\left({ }^{\circ} \mathrm{C}\right)$ \\
Surface Range \\
\end{tabular} & $\begin{array}{c}\text { Monthly Mean } \\
\text { Temperature }\left({ }^{\circ} \mathrm{C}\right) \\
\text { l000M } \\
\end{array}$ & $\begin{array}{c}\text { Annual } \\
\text { Mean } \\
\Delta \mathrm{T}\left({ }^{\circ} \mathrm{C}\right) 500 \mathrm{M} \\
\end{array}$ & $\begin{array}{c}\text { Annual } \\
\text { Mean } \\
\Delta T\left({ }^{\circ} \mathrm{C}\right) \quad 1000 \mathrm{M} \\
\end{array}$ & $\begin{array}{c}\text { Coldest Monthly } \\
\text { Mean } \Delta \mathrm{T}\left({ }^{\circ} \mathrm{C}\right) \\
500 \mathrm{M} \\
\end{array}$ & $\begin{array}{c}\text { Coldest Monthly } \\
\text { Mean } \Delta \mathrm{T}\left({ }^{\circ} \mathrm{C}\right) \\
1000 \mathrm{M} \\
\end{array}$ \\
\hline Sri Lanka & $27.5-28.6$ & $6.5-6.7$ & 18.0 & 21.3 & 17.5 & 20.8 \\
\hline Momb̈asa $\mathrm{N}$ & $25.4-28.7$ & $7.2-7.5$ & 17.0 & 19.5 & 15.6 & 18.2 \\
\hline Mombasa S & $25.5-28.4$ & $6.3-7.0$ & 18.0 & 20.2 & 15.9 & 18.5 \\
\hline Jakarta & $27.2-29.0$ & $5.1-5.6$ & 19.1 & 22.8 & 17.9 & 21.9 \\
\hline Damつier Land & $25.6-28.2$ & $4.9-5.0$ & 19.1 & 22.6 & 17.4 & 20.7 \\
\hline Manila & $27.1-29.5$ & $4.4-4.6$ & 20.0 & $24.0^{\circ}$ & 18.6 & 22.6 \\
\hline Guam & $27.7-29.2$ & $4.3-4.4$ & 21.1 & 24.1 & 20.4 & 23.4 \\
\hline Off Mexico & $22.5-28.0$ & $4.4-4.5$ & 17.6 & 20.9 & 14.9 & 18.0 \\
\hline $\begin{array}{c}\text { Plant Ship } \\
\text { Pacific }\end{array}$ & $27.1-28.5$ & $4.6-4.8$ & 19.4 & 22.8 & 18.1 & 21.7 \\
\hline Ivory Coast & $24.3-28.1$ & 4.5 & 19.2 & 22.1 & 16.8 & 19.7 \\
\hline $\begin{array}{l}\text { Plant Ship } \\
\text { Caribbean }\end{array}$ & $26.4-28.4$ & $5.0-5.3$ & 18.1 & 22.4 & 17.2 & 21.3 \\
\hline
\end{tabular}


SUMMARY OF. SITE PARAMETERS

\begin{tabular}{|c|c|c|c|c|c|}
\hline SITE & $\begin{array}{l}\text { RANGE DISTANCE } \\
\text { (IN KILOMETERS ) } \\
\text { TO SHORE FRCM } \\
\text { l.000 METERS }\end{array}$ & $\begin{array}{l}\text { MONTHLY MEAN } \\
\text { MIXED LAYER } \\
\text { DEPTH METERS } \\
\end{array}$ & $\begin{array}{l}\text { MONTHLY MEAN } \\
\text { SURFACE CURRENTS } \\
(\text { CM/SEC) }\end{array}$ & $\begin{array}{ll}\text { SEA STATE } & \text { MAX } \\
\text { \& OF TIME } & >3 \\
\text { METERS } & \end{array}$ & $\begin{array}{l}\text { NUMBER TROPICAL } \\
\text { CYCLONES PER } \\
\text { YEAR }\end{array}$ \\
\hline SRI LANKA & $22-55$ & $310-30$ & $25-62$ & 3 & $0.2-1.2$ \\
\hline MOMBASA & $33-130$ & $310-90$ & $30-62$ & 2 & $0.0-0.1$ \\
\hline JAKARTA & $18-60$ & $5.5-30$ & $25-52$ & 2 & $0.0-0.1$ \\
\hline DAMPIER LAND & $265-417$ & $3 \cdot 1]-30$ & $25-47$ & 4 & $0.4-1.2$ \\
\hline MANILA & $6-82$ & $20-30$ & $30-52$ & 5 & $4.0-6.0$ \\
\hline GUAM & $7-18$ & $60-120$ & $30-47$ & 5 & $2.0-3.0$ \\
\hline OFF MEXICO & $5-104$ & $10-30$ & $25-31$ & 2 & $0.6-4.0$ \\
\hline $\begin{array}{l}\text { PLANT SHIP } \\
\text { PACIFIC }\end{array}$ & - & $0-30$ & $30-52$ & 2 & $0.0-2.0$ \\
\hline IVORY COAST & $33-52$ & $0-30$ & $25-31$ & 2 & $0.0-0.1$ \\
\hline $\begin{array}{l}\text { PLANT SHIP } \\
\text { CARIBBEAN }\end{array}$ & - & $40-110$ & $30-62$ & 3 & $0.6-1.2$ \\
\hline
\end{tabular}


An examination of the historical oceanographic data for the waters off Manila, Philippines shows that there is an excellent thermal difference resource for OTEC purposes. The region examined was between $14^{\circ} \mathrm{N}-16^{\circ} \mathrm{N}$ latitude and $118^{\circ} \mathrm{E}-120^{\circ} \mathrm{E}$ longitude, which is off the west coast of the island of Luzon. Surface temperatures are high the year round, with the lowest monthly mean surface temperature being $27.2^{\circ} \mathrm{C}$. The average of the monthly mean $\Delta \mathrm{Ts}$ to 1000 meters is $24.0^{\circ} \mathrm{C}$. At 500 meters, the annual thermal difference resource is $20.0^{\circ} \mathrm{C}$, so that the cold water pipes can be relatively short.

The continental slope off the Philippines is steep, and water depths of 1000 meters 1 ie within $18.5 \mathrm{~km}$ (ten nautical miles) off the shores of Luzon. There is an adequate upper mixed layer throughout the year: Typhoons are a major problem for this area. High winds and storms are a bigger problem for this site than any other site studied under the current contract. Seismic activity also occurs. Currents are generally moderate with some variation during the year. 
II. BATHYMETRY

The location of the area of interest off Manila is shown in Figure II-1 (the thermal resource was examined off the Philippine West Coast between 118-120 $\mathrm{E}$ ). Figure II-2 shows the generalized bathymetry around the area to depths of 1500 meters (taken from the U.S. Naval Oceanographic Office Bathymetric Atlas, [40]. The deep Philippine Trench off the east coast and the Manila Trench off the west coast contribute to the high seismic activity in this region.

The width of the continental shelf off Manila varies considerably because of the wide, shallow waters of Manila Bay. Outside the bay, the slope is steep into the Manila Trench. Table II-l summarizes distances to selected depths for several locations. 


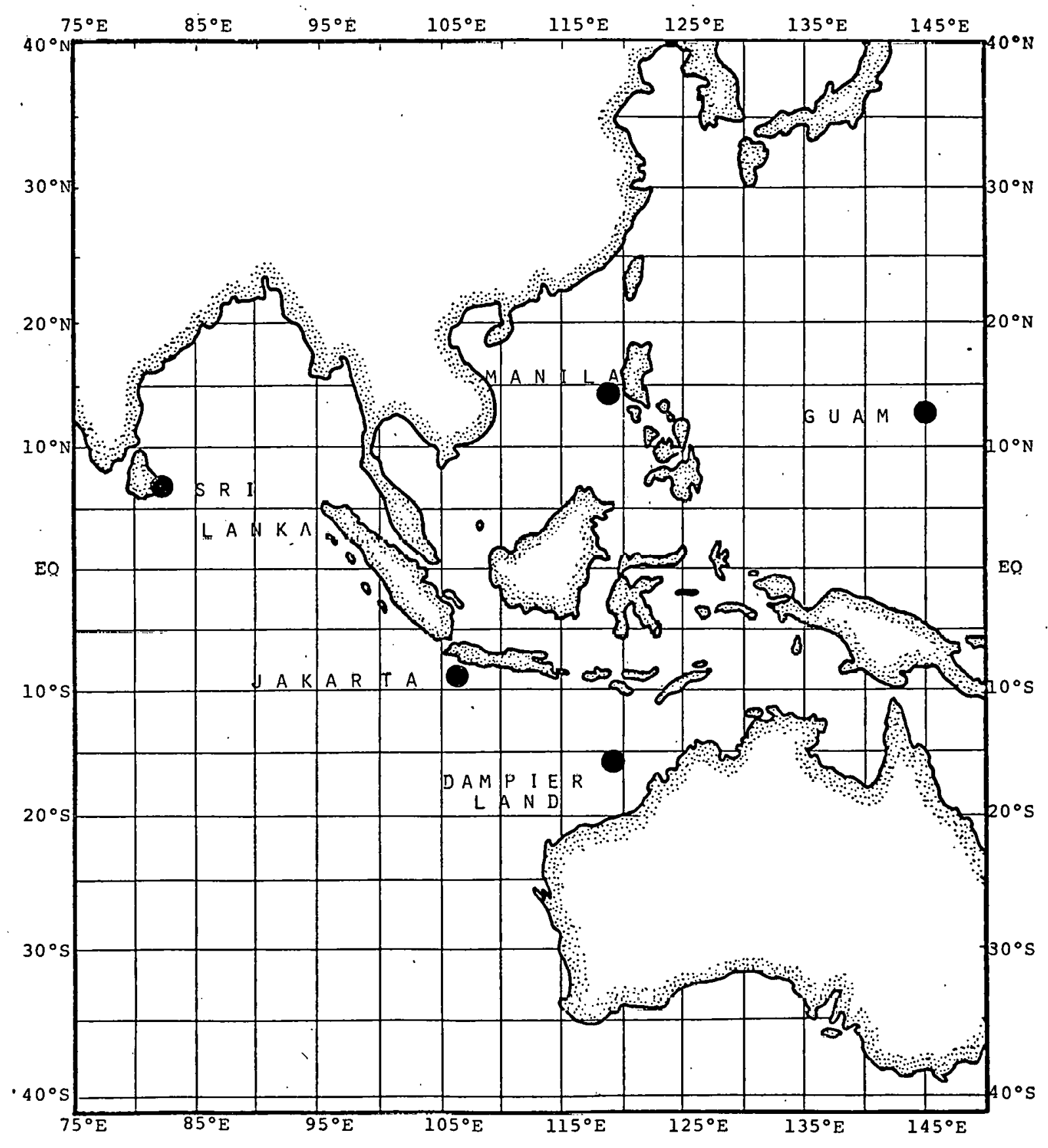

FIGURE II-I: LOCATOR CHART SHOWING. AREA OF RESOURCE INVESTIGATION OFF MANILA. 


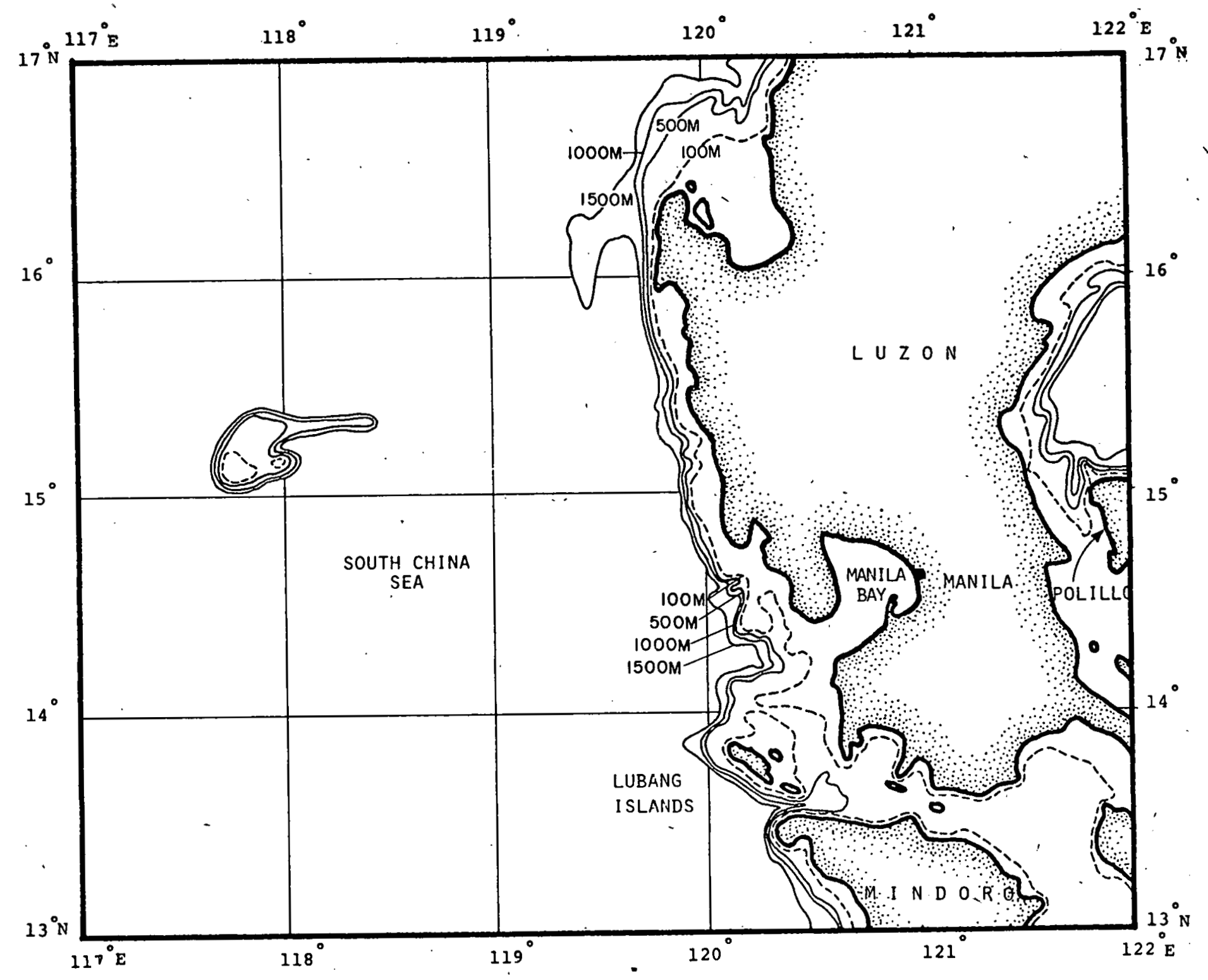

FIGLRE II-2: CHART SHOWING ROUGH BATHYMETRY AROUND LUZON TO DEPTHS OF 1500 METERS. From U.S. Naval Oceanographic Office [40]. 
TABLE II-1: DISTANCES TO SELECTED DEPTHS FOR LOCATIONS AROUND MANILA. From U.S. Naval Oceanographic Office, [40].

1. COASTAL AREAS $14-16^{\circ} \mathrm{N}$

\begin{tabular}{|c|c|c|}
\hline $\begin{array}{c}\text { DEPTH } \\
\text { (Meters) }\end{array}$ & $\begin{array}{c}\text { CLOSEST DISTANCE } \\
\text { (Kilometers) }\end{array}$ & $\begin{array}{c}\text { FARTHEST DISTANCE } \\
\text { (Kilometers) }\end{array}$ \\
\hline 100 & 1.8 & 14.8 \\
500 & 3.7 & 16.6 \\
1000 & 6.5 & 20.4 \\
1500 & 7.4 & 24.0 \\
\hline
\end{tabular}

2. CITY OF MANILA

\begin{tabular}{|c|c|c|}
\hline $\begin{array}{c}\text { DEPTII } \\
\text { (Meters) }\end{array}$ & $\begin{array}{c}\text { CLOSEST DISTANCE } \\
\text { (Kilometers) }\end{array}$ & $\begin{array}{c}\text { FARTHEST DISTANCE } \\
\text { (Kilometers) }\end{array}$ \\
\hline 100 & 70.4 & - \\
500 & 79.6 & - \\
1000 & 81.5 & \\
1500 & 87.1 & \\
\hline
\end{tabular}


Ocean Data System's master subsurface data file is based primarily on soundings from NOAA's National Oceanographic Data Center and the U.S. Navy's Fleet Numerical Weather Central; however, soundings from sources such as foreign and U.S. research activities are inclúded whenever available. In the area off Manila, there was good data coverage to the 500 meter level for every month. Below 500 meters, data were less dense and even completely missing for some months. There were some observations available at 1000 meters from both the winter and summer seasons, and they indicated little annual variation at that depth. Short period fluctuations at all levels were removed using a filtering process described by Wolff, et al (1977), [44].

The thermál resource off Manila is excellent. There is an unlimited supply of cold water available. The warm water supply is also excellent, thus making plant operation possible all year. Monthly mèan surface temperatures range between $27.1^{\circ} \mathrm{C}$ to $29.5^{\circ} \mathrm{C}$. Temperatures below 500 meters do not vary greatly. The temperature difference resource is consistently available. A $\Delta \mathrm{T}$ greater than $20^{\circ} \mathrm{C}$ is available at 550 meters.

Table III-l shows the monthly most probable temperatures off Manila as a function of depth. Table III-2 shows the same data tabulated in terms of $\Delta \mathrm{T}$ (surface temperature minus the temperature at depth) while Figure III-1 shows monthly $\Delta \mathrm{T}$ contours for the area west of Manila. 
TABLE III-1: MONTHLY MOST PROBABLE TEMPERATURE $\left({ }^{\circ} \mathrm{C}\right)$ PROFILE MANILA $14-16^{\circ} \mathrm{N} / 118-120^{\circ} \mathrm{E}$

\begin{tabular}{|c|c|c|c|c|c|c|c|c|c|c|c|c|c|}
\hline \multirow[t]{2}{*}{ Depth } & \multicolumn{11}{|c|}{ Mon th } & \multicolumn{2}{|c|}{$\begin{array}{l}\text { Annual } \\
\text { Mean }\end{array}$} \\
\hline & 1 & 2 & 3 & 4 & 5 & 6 & 7 & 8 & 9 & 10 & 11 & 12 & \\
\hline 0 & 27.2 & 27.1 & 27.6 & 28.6 & 29.4 & 29.5 & 29.1 & 28.9 & 28.9 & 28.8 & 28.4 & 27.7 & 28.4 \\
\hline 50 & 26.8 & 26.2 & 26.0 & 26.2 & 26.8 & 27.5 & 27.7 & 28.0 & 28.0 & 28.0 & 27.7 & 27.3 & 27.2 \\
\hline 100 & 21.8 & 21.5 & 21.4 & 21.4 & 21.8 & 22.4 & 22.7 & 23.3 & 23.4 & 23.3 & 22.6 & 22.6 & 22.3 \\
\hline 150 & 17.5 & .17 .4 & 17.5 & 17.6 & 17.8 & 18.1 & 18.2 & 18.4 & 18.2 & 18.0 & 17.7 & 17.5 & 17.8 \\
\hline 200 & 15.0 & 15.1 & 15.1 & 15.2 & 15.3 & 15.3 & 15.3 & 15.3 & 15.2 & 15.2 & 15.0 & 15.0 & 15.2 \\
\hline 250 & 13.2 & 13.3 & 13.4 & 13.4 & 13.5 & 13.4 & 13.3 & 13.3 & 13.3 & 13.2 & 13.1 & 13.2 & 13.3 \\
\hline 300 & 11.8 & 11.9 & 12.0 & 12.1 & 12.1 & 12.0 & 11.9 & 11.9 & 11.9 & 11.8 & 11.8 & 11.8 & 11.9 \\
\hline 350 & 10.5 & 10.7 & 10.8 & 10.9 & 10.9 & 10.8 & 10.7 & 10.7 & 10.7 & 10.7 & 10.7 & 10.5 & 10.7 \\
\hline 400 & 9.8 & 9.8 & 9.9 & 10.0 & 9.9 & 9.8 & 9.7 & 9.8 & 9.8 & 9.8 & 9.5 & 9.5 & 9.8 \\
\hline 450 & 8.7 & 9.0 & 9.1 & 9.2 & 9.2 & 9.1 & 9.0 & 9.1 & 9.1 & 9.1 & 8.7 & 8.7 & 9.0 \\
\hline 500 & 8.1 & 8.5 & 8.5 & 8.6 & 8.7 & 8.7 & 8.6 & 8.5 & 8.5 & 8.5 & 8.5 & 7.9 & 8.5 \\
\hline 550 & 7.5 & 7.7 & 7.8 & 8.1 & 8.3 & 8.3 & 8.2 & 8.1 & 8.1 & 8.0 & 7.7 & 7.5 & 7.9 \\
\hline 600 & 7.1 & 7.2 & 7.3 & 7.6 & 7.8 & 7.9 & 7.8 & 7.7 & 7.7 & $7.6^{\circ}$ & 7.4 & 7.2 & 7.5 \\
\hline 650 & 6.8 & 6.8 & 6.9 & 7.1 & 7.4 & 7.6 & 7.4 & 7.2 & 7.1 & 7.1 & 7.0 & 6.8 & 7.1 \\
\hline 700 & 6.3 & 6.5 & 6.6 & 6.8 & 7.0 & 7.1 & 6.9 & 6.7 & 6.6 & 6.6 & 6.5 & 6.4 & 6.7 \\
\hline 750 & 6.0 & 6.1 & 6.1 & 6.2 & 6.2 & 6.1 & 6.1 & 6.1 & 6.1 & 6.1 & 6.0 & 6.0 & 6.1 \\
\hline 800 & 5.6 & 5.6 & 5.7 & 5.8 & 5.8 & 5.7 & 5.7 & 5.7 & 5.7 & 5.7 & 5.6 & 5.6 & 5.7 \\
\hline 850 & 5.2 & 5.3 & 5.4 & 5.4 & 5.4 & 5.3 & 5.3 & 5.3 & 5.3 & 5.3 & 5.2 & 5.2 & 5.3 \\
\hline 900 & 4.9 & 5.0 & 5.1 & 5.1 & 5.1 & 5.0 & 5,0 & 5.0 & 5.0 & 5.1 & -5.1 & 4.9 & 5.0 \\
\hline 950 & 4.7 & 4.8 & 4.8 & 4.8 & 4.7 & 4.7 & 4.7 & 4.7 & 4.8 & 4.8 & 4.7 & 4.7 & 4.7 \\
\hline 1000 & 4.4 & 4.5 & 4.5 & 4.5 & 4.4 & $4.4^{\circ}$ & 4.4 & 4.5 & 4.5 & 4.6 & 4.5 & 4.5 & 4.5 \\
\hline 1050 & 4.2 & 4.2 & 4.2 & 4.2 & 4.2 & 4.2 & 4.2 & 4.3 & 4.3 & 4.3 & 4.2 & 4.2 & 4.2 \\
\hline 1100 & 4.0. & 4.0 & 4.0 & 4.0 & 4.0 & 4.0 & 4.0 & 4.0 & 4.0 & 4.0 & 4.0 & 4.0 & 4.0 \\
\hline 1150 & 3.8 & 3.8 & 3.8 & 3.8 & 3.8 & 3.8 & 3.8 & 3.8 & 3.8 & 3.8 & 3.8 & 3.7 & 3.8 \\
\hline 1200 & 3.7 & 3.7 & 3.7 & 3.7 & 3.7 & 3.6 & 3.6 & 3.6 & 3.7 & 3.7 & 3.7 & 3.7 & 3.7 \\
\hline 1250 & 3.5 & 3.5 & 3.5 & 3.5 & 3.5 & 3.5 & 3.5 & 3.5 & 3.5 & 3.5 & 3.5 & 3.5 & 3.5 \\
\hline 1300 & 3.4 & 3.4 & 3.3 & 3.3 & 3.3 & 3.3 & 3.3 & 3,3 & 3.3 & 3.3 & 3.3 & 3.3 & 3.3 \\
\hline 1350 & 3.2 & 3.2 & 3.2 & 3.2 & 3.2 & 3.2 & 3.2 & 3.2 & 3.2 & 3.2 & 3.2 & 3.2 & 3.2 \\
\hline 1400 & 3.1 & 3.1 & 3.1 & 3.1 & 3.1 & 3.1 & 3.1 & 3.1 & 3.1 & 3.1 & 3.1 & 3.1 & 3.1 \\
\hline 1450 & 3.0 & 3.0 & 3.0 & 3.0 & 3.0 & 3.0 & 3.0 & 3.0 & 3.0 & 3.0 & 3.0 & 3.0 & 3.0 \\
\hline 1500 & 3.0 & 3.0 & 2.9 & 2.9 & 2.9 & 2.9 & 2.9 & 2.9 & 2.9 & 2.9 & 2.9 & 2.9 & 2.9 \\
\hline
\end{tabular}


TABLE III-2: SURFACE TEMPERATURE - TEMPERATURE AT DEPTH DIFFERENTIAL $\left({ }^{\circ} \mathrm{C}\right)$ BY MONTHS MANILA $14-16^{\circ} \mathrm{N} / 118-120^{\circ} \mathrm{E}$

\begin{tabular}{|c|c|c|c|c|c|c|c|c|c|c|c|c|c|}
\hline Depth & 1 & 2 & 3 & 4 & 5 & $\begin{array}{c}\text { Month } \\
6\end{array}$ & 7 & 8 & 9 & 10 & 1 & 12 & Annual \\
\hline 50 & 0.4 & 0.9 & 1.6 & 2.4 & 2.6 & 2.0 & 1.4 & 0.9 & 0.9 & 0.8 & 0.7 & 0.4 & 1.3 \\
\hline 100 & 5.4 & 5.6 & 6.2 & 7.2 & 7.6 & 7.1 & 6.4 & 5.6 & 5.5 & 5.5 & 5.8 & 5.1 & $7.3^{\prime}$ \\
\hline 150 & 9.7 & 9.7 & 10.2 & 11.0 & .11 .6 & 11.4 & 10.9 & 10.5 & 10.7 & 10.8 & 10.7 & 10.2 & 10.6 \\
\hline 200 & 12.2 & 12.0 & 12.5 & 13.4 & 14.1 & 14.2 & 13.8 & 13.6 & 13.7 & 13.6 & 13.4 & 12.7 & 13.3 \\
\hline 250 & 14.0 & 13.8 & 14.2 & 15.2 & 15.9 & 16.1 & 15.8 & 15.6 & 15.6 & 15.6 & 15.3 & 14.5 & 15.1 \\
\hline 300 & 15.4 & 15.2 & 15.6 & 16.5 & 17.3 & 17.5 & 17.2 & 17.0 & 17.0 & 17.0 & 16.6 & 15.9 & 16.5 \\
\hline 350 & 16.7 & 16.4 & 16.8 & 17.7 & 18.5 & 18.7 & 18.4 & 18.2 & 18.2 & 18.4 & $17.7^{\circ}$ & 17.2 & 17.7 \\
\hline 400 & 17.4 & 1.7 .3 & 17.7 & 18.6 & 19.5 & 19.7 & 19.4 & 19.1 & 19.1 & 19.0 & 18.9 & 18.2 & 18.7 \\
\hline 450 & 18.5 & 18.1 & 18.5 & 19.4 & 20.2 & 20.4 & 20.1 & 19.8 & 19.8 & 19.7 & 19.7 & 19.0 & 19.4 \\
\hline 500 & 19.1 & 18.6 & 19.1 & 20.0 & 20.7 & 20.8 & 20 . & 20.4 & 20.4 & 20.3 & 19.9 & 19.8 & 20.0 \\
\hline 550 & 19.7 & 19.4 & 19.8 & 20.5 & 21.1 & 21.2 & 20.9 & 20.8 & 20.8 & 20.8 & 20.7 & 20.2 & 20.5 \\
\hline 600 & 20.1 & 19.9 & 20.3 & 21.0 & 21.6 & 21.6 & 21.3 & 21.2 & 21.2 & 21.2 & 21.0 & 20.5 & 20.9 \\
\hline 650 & 20.4 & 20.3 & 20.7 & 21.5 & 22.0 & 21.9 & 21.7 & 21.7 & 21.8 & 21.7 & 21.4 & 20.9 & 21.3 \\
\hline 700 & 20.9 & 20.6 & 21.0 & 21.8 & 22.4 & 22.4 & 22.2 & 22.2 & 22.3 & 22.2 & 21. & 21.3 & 21.8 \\
\hline 750 & 21.2 & 21.0 & 21.5 & 22.4 & 23.2 & 23.4 & 23.0 & 22.8 & 22.8 & 22.7 & 22.4 & 21.7 & 22.3 \\
\hline 800 & 21.6 & 21.5 & 21.9 & 22.8 & 23.6 & 23.8 & 23.4 & 23.2 & 23.2 & 23.1 & 22.8 & 22.1 & 22.8 \\
\hline 850 & 22.0 & 21.8 & 22.2 & 23.2 & 2 & 24.2 & 23 & 2 & 23 & 23.5 & 23 & 22.5 & 23.1 \\
\hline 900 & 22.3 & 22.1 & 22.5 & 23.5 & 24.3 & 24.5 & 24.1 & 23.9 & 23.9 & 23.7 & 23.3 & 22.8 & 23.4 \\
\hline 950 & 22.5 & 22.3 & 22.8 & 23.8 & 24.7 & 24.8 & 24.4 & 24.2 & 24.1 & 24.0 & 23.2 & 23.0 & 23.7 \\
\hline 1000 & 22.8 & 22.6 & 23.1 & 24.1 & 25.0 & 25.1 & 24.7 & 24.4 & 24.4 & 24.2 & 23.9 & 23.2 & 24.0 \\
\hline 1050 & 23.0 & 22.9 & 23.4 & 24.4 & 25.2 & 25.3 & 24.9 & 24.6 & 24.6 & 24.5 & 24.2 & 23.5 & 24.2 \\
\hline 1100 & 23.2 & 23.1 & 23.6 & 24.6 & 25.4 & 25.5 & 25.1 & 24.9 & 24.9 & 24.8 & 24.4 & 23.7 & 24.4 \\
\hline 1150 & 23.4 & 23.3 & 23.8 & 24.8 & 25.6 & 25.7 & 25.3 & 25.1 & 25.1 & 25.0 & 24.6 & 24.0 & 24.6 \\
\hline 1200 & 23.5 & 23.4 & 23.9 & 24.9 & 25.7 & 25.9 & 25.5 & 25.3 & 25.2 & 25.1 & 24.7 & 24.0 & 24.8 \\
\hline 1250 & 23.7 & 23.6 & 24.1 & 25.1 & 25.9 & 26.0 & 25.6 & 25.4 & 25.4 & 25.3 & 24.9 & 24.2 & 24.9 \\
\hline 1300 & 23.8 & 23.7 & 24.3 & 25.3 & 26.1 & 26.2 & 25.8 & 25.6 & 25.6 & 25.5 & 25.1 & 24.4 & 25.1 \\
\hline 1350 & 24.0 & 23.9 & 24.4 & 25.4 & 26.2 & 26.3 & 25.9 & 25.7 & 25.7 & 25.6 & 25.2 & 24.5 & 25.2 \\
\hline 1400 & 24.1 & 24.0 & 24.5 & 25.5 & 26.3 & 26.4 & 26.0 & 25.8 & 25.8 & 25.7 & 25.3 & 24.6 & 25.3 \\
\hline 1450 & 24.2 & 24.1 & 24.6 & 25.6 & 26.4 & 26.5 & 26.1 & 25.9 & 25.9 & 25.8 & 25.4 & 24.7 & 25.4 \\
\hline 1500 & 24.2 & 24.1 & 24.7 & 25.7 & 26.5 & 26.6 & 26.2 & 26.0 & 26.0 & 25.9 & 25.5 & 24.8 & 25.5 \\
\hline
\end{tabular}




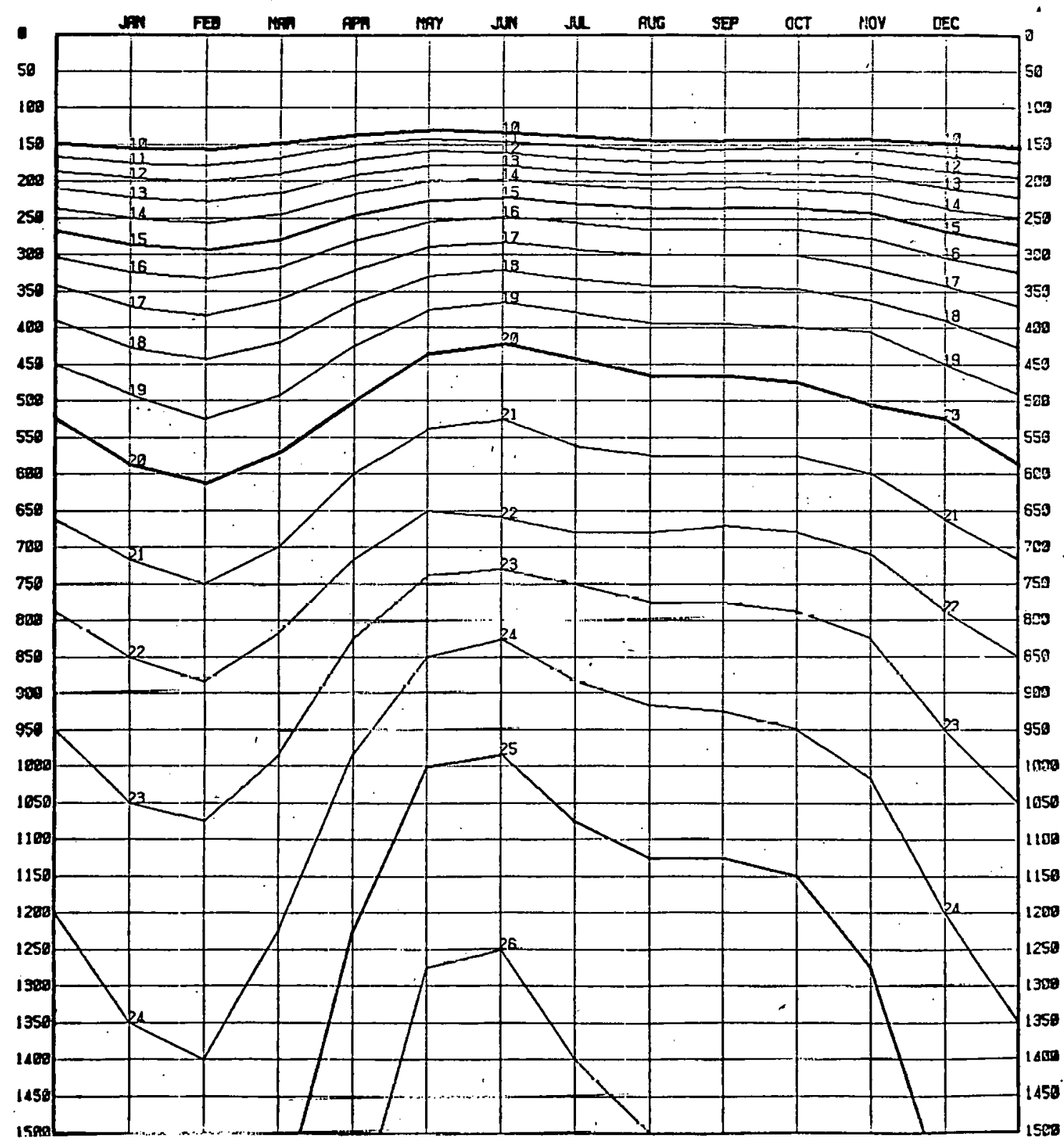

FIGURE III-1: CHART SHOWING MONTHLY $\triangle T$ TON'IUURS $\left({ }^{\circ} \mathrm{C}\right)$ AT A DFPTH RESOLIITION OF 50 ME'L'LRS FOR A $2^{\circ}$ LAT/LONG RECTANGLE WEST OF MANILA. 
IV. MIXED LAYER DEPTH

For OTEC purposes, the depth of the upper mixed layer is defined as the depth at which the temperature is first $1^{\circ} \mathrm{C}$ colder than the sea surface temperature. A well defined and moderately deep upper mixed layer is favorable for OTEC . operations. Off Manila, a mixed layer is always present but it is not very deep -- particularly from March to June. The upper thermal structure off Manila should be adequate for OTEC operations, but it is not ideal.

Typical monthly MLD values for this area are shown in Table IV-1. They were determined: from analysis of the thermal resource data (see section III).

TABLE IV-1: TYPICAL MIXED LAYER DEPTHS (METERS) WEST OF MANIIAA.

\begin{tabular}{|cccccc|}
$\frac{\text { JAN }}{60}$ & $\frac{\text { FEB }}{60}$ & $\frac{\text { MAR }}{30}$ & $\frac{\text { APR }}{20}$ & $\frac{\text { MAY }}{20}$ & $\frac{\text { JUN }}{30}$ \\
$\frac{\text { JUL }}{50}$ & $\frac{\text { AUG }}{50}$ & $\frac{\text { SEP }}{60}$ & $\frac{\text { OCT }}{45}$ & $\frac{\text { NOV }}{60}$ & $\frac{\text { DEC }}{80}$ \\
\hline
\end{tabular}


V. WEATHER CONDITIONS

The Manila site is in the center of a major typhoon track as shown in Figure V-I from Crutcher and suayle (1974), []]. Typhoons have passed through this area during all months of the year but are most common from October through December. The typical typhoon count per 10-year period as reported by Crutcher and Quayle is shown in Table V-l.

TABLE V-l: AVERAGE TYPHOON COUNT PER TEN YEAR PERIOD IN THE MANILA AREA. From Crutcher and Quayle (1974), [I].

\begin{tabular}{|cccccc}
\hline$\frac{\text { JAN }}{0}$ & $\frac{\text { FEB }}{0}$ & $\frac{\text { MAR }}{1}$ & $\frac{\text { APR }}{1}$ & $\frac{M A Y}{2}$ & $\frac{\text { JUN }}{2}$ \\
$\frac{\text { JUL }}{2}$ & $\frac{\text { AUG }}{2}$ & $\frac{\text { SEP }}{2}$ & $\frac{\text { OCT }}{4}$ & $\frac{\text { NOV }}{5}$ & $\frac{\text { DEC }}{3}$ \\
\hline
\end{tabular}

OTEC plants in the area off Manila must be able to withstand typhopon winds in excess of 70 meters/second. Mountainous waves can be produced. Gentry (1970), [18] states that occasional waves of over twenty meters have been reported in association with hurricanes and that there are numerous reports of waves 10-15 meters. A NDDBO study (1978), [20], indicates that hurricanes can cause a temperature anomaly - of $-3^{\circ} \mathrm{C}$ for a period of four days. While this result was measurner i.n the Gulf of Mexico, it should be indicative of the effects of hurricanes on the thermal resource anywhere in the tropics, including the waters west of Manila. 
Table V-2, from the U.S. Naval Weather Service (1983), [36], summarizes tropical storm statistics for the Manila area; these statistics include typhoons and tropical storms of lesser intensity.

Table V-3 from the U.S. Naval Weather Service (1938), [31], summarizes prevailing wind direction and speed by months for the area off Manila. These values are representative only of the basic flow in the area; planners must be careful to account for the relatively frequent tropical storms and typhoons which are embedded in this basic flow. 


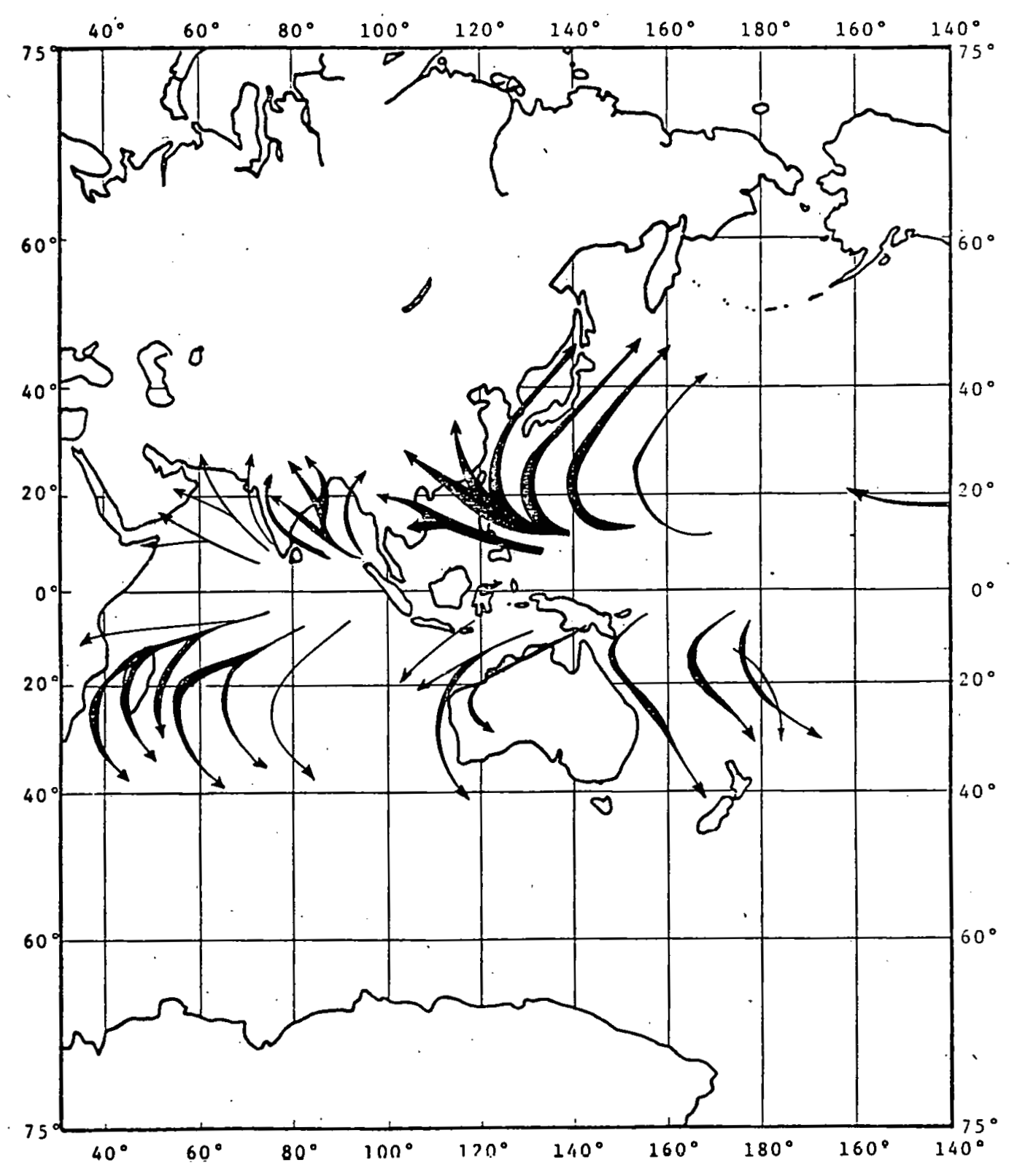

FIGURE V-I: ANNUAL PREFERRED STORM TRACKS FOR TROPICAL STORMS SHOWING THAT THE MANILA SITE TS YARTICULARLY EXPOSED. 
TABLE $v-2:$ TROPICAL STORM TRACK STATISTICS FOR THE MANILA AREA. From U.S. Naval weather Service (1973), [36].

\begin{tabular}{|c|c|c|c|c|c|c|c|c|c|c|c|c|}
\hline \multirow[b]{2}{*}{ PARAMETER } & \multicolumn{12}{|c|}{ M.DNTH } \\
\hline & JAN & FEB & MAR & APR & MAY & JUN & JUL & AUG & SEP & OCT & NOV & $\mathrm{DEC}$ \\
\hline $\begin{array}{l}\text { 1. MOST PROBABLE } \\
\text { DIRECTION OF MOVE- } \\
\text { MENT (TOward) }\end{array}$ & $W$ & WNW & WNW & WNW & WNWW & WNh & 'NNW & WNW & WNW & WNW & W & $W$ \\
\hline $\begin{array}{l}\text { 2. LIKELIHOOD STORM WILL } \\
\text { MOVE THIS DIRECTION } \\
\text { (Percent). }\end{array}$ & 98 & 72 & 87 & 85 & 82 & 92 & 93 & 93 & 92 & 83 & 77 & 76 \\
\hline $\begin{array}{l}\text { 3. PROBABLE LATERAL } \\
\text { SPEED CF MOVEMENT } \\
\text { (M/S) }\end{array}$ & 5 & 5 & 5 & 4.5 & 4.5 & 4.5 & 5.5 & 5.5 & 5.5 & 4.5 & 5 & 4.5 \\
\hline $\begin{array}{l}\text { 4. NUMBER OF STORMS OR } \\
\text { CYCLGNES PER YEAR } \\
\text { PER } 5^{\circ} \text { SQUARE }\end{array}$ & 0.08 & 0.10 & 0.05 & 0.15 & 0.25 & 0.39 & 0.42 & 0.51 & 0.53 & 0.57 & 0.74 & 0.27 \\
\hline
\end{tabular}


TABLE V-E: WIND FLOW STATISTICS FOR THE AREA WEST OF MANILA. BASIC FLOW ONLY. From U.S. Naval Weather Service (1973), [36].

\begin{tabular}{|c|c|c|c|c|c|c|c|c|c|c|c|c|}
\hline \multirow[b]{2}{*}{ FARAMETER } & \multicolumn{12}{|c|}{-MONTH } \\
\hline & JAN & FEB & MAR & APR & MAY & JUN & J̦UL & AUG & SEP & $\mathrm{OCT}$ & NOV & $\mathrm{DEC}$ \\
\hline $\begin{array}{l}\text { 1. PREVAILING WINL } \\
\text { DIRECTION }\end{array}$ & NNE & NNE & NNE & $\mathrm{NE}$ & NE & $S$ & SSW & SW & SW & VAR & NNE & $\mathrm{NE}$ \\
\hline $\begin{array}{l}\text { 2. MEAN WIND SPEED } \\
(\mathrm{M} / \mathrm{S})\end{array}$ & 7.7 & 6.2 & 6.2 & 5.1 & 3.1 & 2.1 & 2.6 & 5.7 & 4.6 & 5.1 & 6.2 & 8.2 \\
\hline
\end{tabular}


Table V-4 summarizes general climatic conditions at Manila bỳ months. The pattern is characteristic of a monsoonal regime with a strong summer maximum of precipitation due to frequent showers and thunderstorms. In spite of the larger number of typhoons late in the year, their contribution to total rainfall is relatively small. 
TABLE V-4: CLIMATIC SUMMARY FOR MANILA, PHILIPPINES (Elevation 25 meters, Latitude $1431 \mathrm{~N}$, Longitude $12101 \mathrm{E})$. From U.S. Naval Weather

Service (1973), [36].

\begin{tabular}{|c|c|c|c|c|c|c|c|c|c|c|c|c|}
\hline \multirow[b]{2}{*}{ PARAMETER } & \multicolumn{12}{|c|}{ MONTH } \\
\hline & JAN & FEB & MAR & APR & MAY & JUN & JUL & AUG & SEP & OCT & NOV & $\mathrm{DEC}$ \\
\hline ABSOLUTE MAX TEMP $\left({ }^{\circ} \mathrm{C}\right)$ & 33 & 33 & 37 & 37 & 40 & 38 & 35 & 34 & 34 & 36 & 34 & 33 \\
\hline AVERAGE MAX TEMP $\left({ }^{\circ} \mathrm{C}\right)$ & 29 & 31 & 32 & 34 & 34 & 33 & 31 & 31 & 32 & 32 & 31 & 30 \\
\hline MEAN TEMP $\left({ }^{\circ} \mathrm{C}\right)$ & 26 & 27 & 28 & 29 & 30 & 29 & 28 & 28 & 28 & 28 & 28 & 27 \\
\hline AVERAGE MIN TEMP $\left({ }^{\circ} \mathrm{C}\right)$ & 22 & 22 & 24 & 26 & 28 & 26 & 26 & 26 & 26 & 26 & 26 & 24 \\
\hline ÄBSOLUTE MIN TEMP $\left({ }^{\circ} \mathrm{C}\right)$ & 20 & 20 & 19 & 21 & 23 & 22 & 21 & 21 & 22 & 22 & 22 & 20 \\
\hline AVERAGE RAINFALL (MM) & 14 & 7 & 6 & 21 & 119 & 257 & 329 & 385 & 352 & 141 & 98 & 31 \\
\hline MEAN NO. DAYS RAIN ' & 5 & 3 & 3 & 4 & 11 & 17 & 22 & 22 & 21 & 17 & 13 & 9 \\
\hline MEAN NO.DAYS THUNDERSTORM & 0 & $<1$ & 1 & 3 & 9 & 11 & 8 & 8 & 8 & 6 & 2 & 1 \\
\hline MEAN NO. DAYS FOG & 0 & 0 & 0 & 0 & 0 & 0 & 2 & 2 & $<1$ & $<1$ & $<1$ & $<1$ \\
\hline MEAN RELATIVE HUMIDITY $(\%)$ & 75 & 72 & 70 & 70 & 70 & 79 & 81 & 83 & 83 & 78 & 77 & 76 \\
\hline PREVAILING WIND DIRECTION & $\mathrm{E}$ & $\mathrm{E}$ & ESE & SE & ESE & W & w & $\mathrm{W}$ & W & $E$ & $\mathrm{E}$ & $\mathrm{E}$ \\
\hline MEAN WIND SPEED (M/S) & 4 & 4 & 6 & 6 & 6 & 4 & 4 & 4 & 4 & 3 & 3 & 4 \\
\hline
\end{tabular}

* Not characteristic of OTEC area west of Manila. 
VI. SEA AND SWELL CONDITIONS

Distribution of sea and swell conditions for a typical year off Manila are as follows:

\begin{tabular}{|c|c|c|}
\hline & $\begin{array}{c}\text { Low Seás } \\
(0.3-0.9 \text { meters })\end{array}$ & $\begin{array}{c}\text { High Seas } \\
\text { meters or higher })\end{array}$ \\
\hline JAN-FEB & $80 \%$ & $0-10 \%$ \\
\hline JUL-AUG & $80 \%$ & $10 \%$ \\
\hline
\end{tabular}

\begin{tabular}{|l|c|c|}
\hline & $\begin{array}{c}\text { Low Swell } \\
(0.3-0.9 \text { meters })\end{array}$ & $\begin{array}{c}\text { High Swell } \\
\text { meters or higher })\end{array}$ \\
\hline JAIN-FEB & $60-80 \%$ & $0-10 \%$ \\
\hline JUL-AUG & $80 \%$ & $0-10 \%$ \\
\hline
\end{tabular}

The source of this information is from Bigelow and Emundson's Wind Waves At Sea, (1947, [ 5 $]$ ).

High swell conditions with wave height in excess of 3.6 meters occurs between $0-2 \%$ of the time in each of the four seasons. Waves qreater than 2.4 meters also only occur between $0-2 \%$ of the time, except in the fall when the frequency is 2-5\%. The northern half of Luzon has a slightly greater frequency of high conditions in the fall and winter. Table VI-I provides wave statistics for the Manila site' taken from Ocean Wave Statistics (1967, [19]). This statistical breakdown repreșents the number of actual observations

$$
\mathrm{VI}-1
$$


TABLE VI-I: STATISTICAL BREAKDOWN SHOWING NUMBER GF SHIPS OBSERVATIONS OFF THE WEST COAST OF LUZON REPORTING VARIOUS HEIGHT/PERIOD COMBINATIONS (ALL SEASONS). From Ocean Wave Statistics (1967),.. [19].

\begin{tabular}{|c|c|c|c|c|c|c|c|c|c|c|c|c|c|}
\hline $\begin{array}{l}\text { Wave Height } \\
\text { (meters) }\end{array}$ & $\mathrm{CaI}$ & $<5$ & $\epsilon-7$ & $\begin{array}{l}\text { Wave } \\
8-9\end{array}$ & $\begin{array}{l}\text { e Perio } \\
10-i i\end{array}$ & $\begin{array}{c}\text { d } \quad \text { Seco } \\
12-13 \\
\end{array}$ & $\begin{array}{l}\text { nas) } \\
14-15\end{array}$ & $16-17$ & $18-19$ & $20-21$ & $\begin{array}{c}\text { over } \\
21\end{array}$ & Total & $\begin{array}{l}\text { Percent of } \\
\text { Grand Total }\end{array}$ \\
\hline 0.25 & $23 h$ & 343 & 7. & 3 & 1 & & & & & 6 & 4 & 598 & 9 \\
\hline 0.5 & 10 & 793 & 54 & 10 & 2 & & I & & & 1 & 28 & 899 & 14 \\
\hline 1.0 & 16 & 1061 & 370 & 47 & 15 & 2 & & 1 & & 4 & 18 & 1534 & 24 \\
\hline 1.5 & 12 & 426 & 612 & 134 & 26 & 5 & 1 & 1 & & & 1 & 1268 & 20 \\
\hline 2.0 & $=$ & 109 & 375 & $2 \geq 4$ & 41 & 9 & $I$ & 1 & & & & 761. & 12 \\
\hline 2.5 & & 47 & 191 & 217 & .82 & 15 & 4 & & & & & 556 & 9 \\
\hline 3.0 & 3 & 16 & 86 & 138 & 77 & 30 & 8 & & & & & 358 & 6 \\
\hline 3.5 & 4 & 7 & 33 & 72 & 49 & 16 & 13 & $I$ & & & & 195 & 3 \\
\hline 4.0 & $\therefore$ & & 10 & 39 & 31 & 11 & 4 & . & & & & 95 & 1 \\
\hline 4.5 & & 3 & 19 & 31 & 30 & 10 & 8 & 1. & & & & 102 & 1 \\
\hline 5.0 & & 1 & & 6 & 11 & 2 & 2 & & & & & 22 & $<1$ \\
\hline 5.5 & $I$ & & & 12 & 4 & 2 & $I$ & & & & & 20 & $<1$ \\
\hline 6.0 & & & & 11 & 11 & 1 & & & & & & 24 & $<1$ \\
\hline 6.5 & & & 3 & 4 & 4 & 1 & 3 & . & & & & 16 & $<1$ \\
\hline 7.0 & & & 1 & 2 & 1 & 1 & & & & . & & 6 & $<1$ \\
\hline 7.5 & 1 & & & 2 & & & & & & & & 3 & $<1$ \\
\hline TOTAL & $28 \tilde{c}$ & 2306 & 1762 & 1002 & 385 & 107 & 46 & 7 & & 12 & 52. & 6461 & \\
\hline $\begin{array}{l}\text { Pexcent } \\
\text { of Grand } \\
\text { Total. }\end{array}$ & 4 & 43 & 27 & 16 & 6 & 2 & $<1$ & $<1$. & $<$ & $<1$ & $<1$ & & \\
\hline
\end{tabular}


in various height versus period categories. The observations are from an area somewhat larger than the area chosen for site analysis, but the conditions over the area are represented as being homogeneous. Figure VI-1 provides a graph of the relative frequency of various wave heights for the Manila site. This site is susceptible to hurricanes so OTEC plants should be built to withstand mountainous waves. Table VI-2 from Climatic Summaries For Major Seventh Fleet Ports and Waters (1973, [36]), provide monthly mean wave heights for this site.

TABLE VI-2: MONTHLY MEAN WAVE HEIGHTS IN METERS FOR MANILA SITE WATERS.

\begin{tabular}{|c|c|c|c|c|c|}
\hline JAN & FEB & MAR & $\underline{A P R}$ & $\underline{M A Y}$ & JUN \\
\hline $1.2-2.1$ & $1.2-2.1$ & $0.6-0.9$ & $0.6-1.5$ & $0.6-0.9$ & $0.6-1.5$ \\
\hline JUL & $\underline{\text { AUG }}$ & SEP & OCT & NOV & $\underline{\mathrm{DEC}}$ \\
\hline $0.6-1.5$ & $0.6-1.5$ & $0.6-1.5$ & $0.6-1.5$ & $1.2-1.5$ & $1.2-1.5$ \\
\hline
\end{tabular}




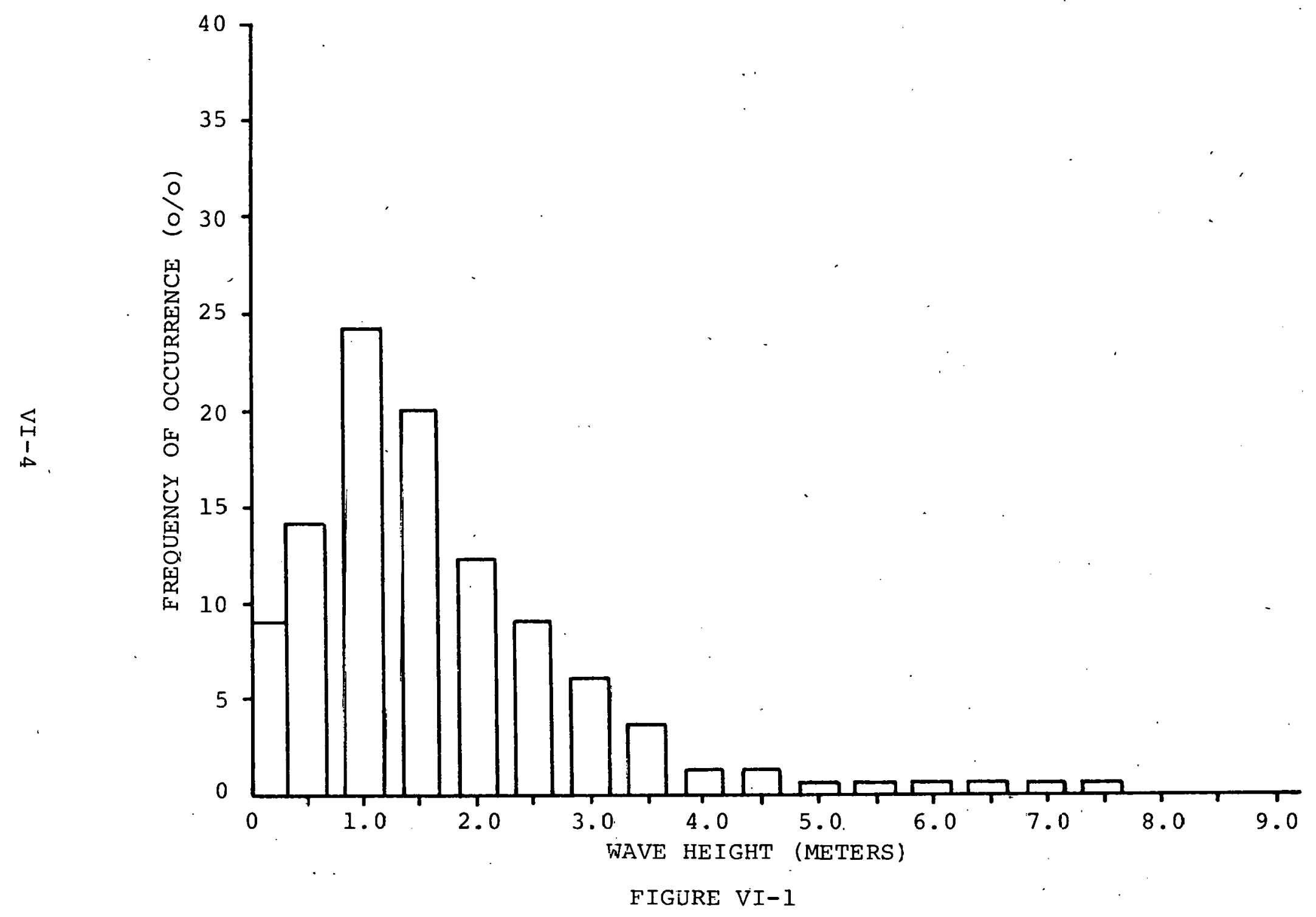

WAVE HEIGHT FREQUENCIES (MANILA) 
VII. CURRENTS

The Northeast Trade winds predominate in this latitude east of the Philippines. As far west as the Philippines, however, the Northwest Tradewind Current and the North Equatorial Current have lost most of their strength. This area off the Philippines, therefore, experiences considerable variability in surface currents. The currents are variable both in speed and direction. Generally, the currents are weak to moderate in speed, with the vast majority of occurrences having a velocity less than $40 \mathrm{~cm} / \mathrm{sec}$. The monthly mean surface current patterns extracted from Defense Mapping Agency Hydrographic Center Pilot Charts (1978, [8]) are:

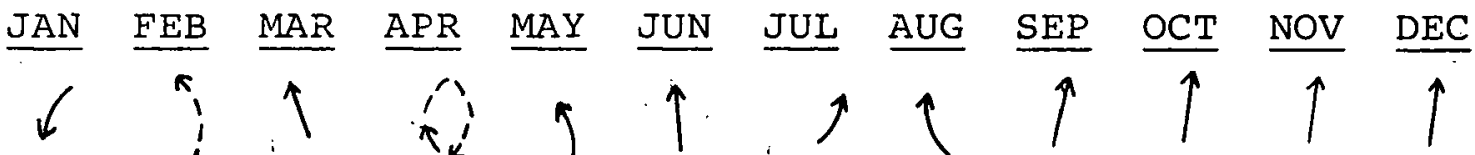
$41.1 \quad 30.8 \quad 36.0 \quad 30.8 \quad 30.8 \quad 41.1 \quad 41.1 \quad 36.0 \quad 36.0 \quad 46.3 \quad 41.141 .1$

Current directions are shown by arrows and current speeds in $\mathrm{cm} / \mathrm{sec}$.

This surface current data is useful as a rough estimate of mean conditions. Pilot charts are based on ship logs which are generally determined in offsets between dead-reckoning fixes and navigational fixes. Few direct measurements of currents are available and very few sub-surface measurements.

These current figures are mean and not indicative of currents when high winds or sea and swell conditions prevail. Hurricanes also effect surface currents. Leipper's (1967 [21]) 
study of Hurricane Hilda showed that a current had developed in the area transversed by the storm. Analysis of temperature depth data showed a current of approximately $50 \mathrm{~cm} / \mathrm{sec}$. A theoretical study by O'Brien and Reid (1967, [27]) states that hurricanes will. cause currents with a speed of approximately one meter per second.

The passage of hurricanes will induce upwelling of subsurface water for a temporary period. This upwelling will cause anomalous vertical current shears. Vertical and horizontal current shears are also observed in association with major current systems. The Philippines, especially the western coast, is removed from any major current system. 
1. Adamec, D., and O'Brien, J.J., November 1978, "The Seasonal Upwelling in the Gulf of Guinea Due to Remote Forcing", J. Phys. Oceanogr., 8, No. 6, 1050-1060.

2. Atwood, D.K., et al., 1976, Ocean Thermal Energy Conversion, Resource Assessment and Environment Impact for Proposed Puerto Rico Site, University of Puerto Rico.

3. Avery, W.H., et al., 1976, Maritime and Construction Aspects of Ocean Thermal Energy Conversion (OTEC) Plant Ships, The John Hopkins University Applied Physics. Laboratory, Laurel, Maryland.

4. Bathen, K.H., et al., 1977, "Consolidated Oceanographic and Meteorological Data for Four North Pacific OTEC Sites", University of Hawaii.

5. Bigelow, H.B., and W.T. Emundson, 1947, Wind Waves at Sea, U.S. Hydrographic Office, Washington, D.C.

6. Colburn, J.G., 1974, "The Thermal Structure of the Indian Ocean", International Indian Ocean Expedition Oceanographic Monograph No., 2; The University Press of Hawaii; Honolulu, Hawaii.

7. Crutcher, H.L. and R.G. Quaýle, 1974, "Mariners Worldwide Climatic Guide to Tropical Storms at Sea", NAVAIR 501C-61, Naval Weather Service; Asheville, N.C.

8. Defense. Mapping Agency Hydrographic/Topographic Center, 1978, Pilot Chart of the North Pacific, DMA Stock Numbers PILOT 557801, 557804, 557810, Washington, D.C.

9. Defense Mapping Agency Hydrographic/Topographic Center, 1978, Pilot Chart of the North Atlantic Ocean, DMA Stock Numbers PILOT 167801, 167804, 167807, 167810, Washington, D.C.

10. Defense Mapping Agency Hydrographic Center, South Pacific Ocean, Sheet.IV, Pub. N.O. 623, revised 1974, Washington, D.C.

11. Defense Mapping Agency Hydrographic Center, Indian Ocean, Northern Part, Pub 1No. 721, 1974, Washington, D.C.

12. Defense Mapping Agency Hydrographic Center, Gulf of Mexico and Carribean Sea, Pub. No. 410, revised 1973, Washington, D.C. 
13. Defense Mapping Agency Hydrographic Center, North Atlantic Ocean, Southeastern Sheet, Publication N.O. 125, 4th ed., 1974, Washington, D.C.

14. Düing, W., 1970; "The Monsoon Regime of the Currents in the Indian Ocean", International Indian Ocean Expedition Oceanographic Monograph No. 1, Hawaii Institute of Geophysics Contribution No. 331, the University of Hawaii Press; Honolulu, Hawaii.

15. Düing, W. and F. Schott; March 1978, "Measurements in the Source Region of the Somali Current during the Monsoon Reversal", J. Phys. Oceanogr., 8, 278-289.

16. Emilsson, J., 1970, "On the Upper Layer Circulation in the Cayman Sea", from the Symposium on the Investigations and Resources of the Caribbean Sea and Adjacent Regions, UNESCO, Paris France.

17. Fleet Numerical Weather Central (FNWC), FNWC and National Oceanographic Data Center digitized reports.

18. Gentry, R.C., 1970, "Hurricanes, One of the Major Features of Air-Sea Interaction in the Caribbean Sea", from the Symposium on the Investigation and Resources of the Caribbean Sea and Adjacent Regions, UNESCO, Paris, France.

19. Hogben, N., and F. Lumb, 1967, Ocean Wave Statistics, National Physical Laboratory, Ministry of Technology, Iondon. Her Majesty's Stationery Office.

20. Johnson, A. and S. Denwick, 1978; "Data Report Buoy Observations During Hurricanes Anita and Babe, AugustSeptember, 1977", NOAA Data Buoy Office, National Space Technology Laboratories, NSTL Stațion; Mississippi.

21. Le1pper, D.F., 1967, "Übserved Uceàn Londitions and Furricane Hilda, 1964. J. Ațmos Sci., 24, p 182-196.

22. MCFadden J.D., "Airborne Investigations of the Effects of Hurricanes on the Thermal structure of the surface Layer of the Ocean", from the Symposium on Investigation and Resources of the Caribbean Sea and Adjacent Regions, UNESCO, Paris France.

23. National Oceanographic Data Center, The Variability of Water Masses in the Indian Ocean, Publication G-11, Washington, D.C. 
24. National Oceanographic Data Center, Data File BTG75A, Washington, D.C., 1977.

25. National Ocèanographic Data Center, Data Files SD40A5/0A6 from SD76A (1/30/76) 3509 STA: Washington, D.C., 1977.

26. National Science Foundation, 1972, Meteorological Atlas of the International Indian Ocean Expedition, Volume 7, Washington, D.C.

27. O'Brien, J.J., and R.O. Reid, 1967, "The Non-Linear Response of a Two Layer Baroclinic Ocean to a Stationery Axially Symetric Hurricane", J. Atmos. Sci., 24, p 197215 .

28. Ramage, C.D., 1972, "Indian Ocean Surface Meteorology", International Indian Ocean Expedition, Collective Reprints VIII, Contribution No. 624, pgs 407-540; Paris, France.

29. Sirvastava, P.S., P.K. Vyayarayou and M.X. Joseph, 1972 "Monthly Wave Characteristics of the Bay of Bengal", International Indian Ocean Expedition, Collective Reprints VIII, Contribution No. 625, Paris, France.

30. The Enclyopedia of Oceanography, 1966, Reinhold Publishing Corp., New York, New York.

31. U.S. Department of Agriculture, Weather Bureau, 1938, "Atlas of Climatic Charts of the Oceans", Washington, D.C.

32. U.S. Department of Commerce, NOAA, 1971, Eastropac Atlas, Volume 3, Washington, D.C.

33. U.S. Navy, 1975, Marine Climatic Atlas of the World, Volume III, Indian Ocean, Washington, D.C.

34. U.S. Navy Hydrographic Office, 1964, Atlas of Sea and Swell Charts, Northeastern Pacific Ocean, Publication No. 799D, revised 1976, Washington, D.C.

35. U.S. Naval Weather Service, September 1974, Climatic Summaries For Major Indian Ocean Ports and Waters, NAVAIR 50-lC-63, Asheville, N.C.

36. U.S. Naval Weather Service, November 1973, Climatic Summaries For Major Seventh Fleet Ports and Waters, NAVAIR 50-lC-62, Asheville, N.C.

37. U.S. Naval Oceanographic Office, 1963, Oceanographic Atlas of the North Atlantic Ocean, Washington, D.C. 
38. U.S. Naval Oceanographic Office, Bathymetric Atlas of the Northeastern Pacific Ocean, Pub No. 1303-S, No. $0902 \mathrm{~N}, 0903 \mathrm{~N}, 1002 \mathrm{~N}, 1003 \mathrm{~N}$, Washington, D.C.

39. U.S. Naval Oceanographic Office, Indian Ocean, AfricaEast Coast Publication No. 724, lst ed., 1974, Washington, D.C.

40. U.S. Naval Oceanographic Office, 1971, Eathymetric Atlas of the Northeastern Pacific Ocean, H.O. Pub No. $1303-\mathrm{S}, \mathrm{N} .0 .1104 \mathrm{~N}, 1105 \mathrm{~N}, 1204 \mathrm{~N}, 1204 \mathrm{~N}$, Washington, D.C.

41. U.S. Naval Oceanographic Office, 1971, Bathymetric Atlas of the Northeastern Pacific Ocean, H.O. Pub N.O. $1301-\mathrm{S}, 2403 \mathrm{~N}, 2040 \mathrm{~N}$, Washington, D.C.

42. U.3. Naval Oceanographic Office, 1970, Balhymelric Atlas of the Northwestern Pacific Ocean, H.O. Pub. $1201-S$, N.O. 2203N, Washington, D.C.

43. Wolff, P.M. and W.E. Hubert, 1976, "Ocean Thermal Linergy Conversion: Resource, Ecological and Environmental Studies", Contract No. NSF-C1020, Ocean Data Systems, Inc., Monterey, California.

44. Wolff, P.M., et al, 1977, "OTEC Resource Report for Hawaii", Contract EG-77-C-01-4028, Ocean Data Systems, Inc., Monterey, California.

45. Wyrtki, K., 1972, "The Upwelling in the Region Between Java and Australia during the South-East Monsoon, Collected reprints of the International Indian Ocean Expedition, Volume I, Contribution No. 15, pp 151-161.

46. Wyrtki, K., 1972, "Geopotential Topographics and Associated Circulation in the South Eastern Indian Ocean", Collected reprints of the International Indian Ocean, Expedition, Volume I, contribution No. 14 .

47. Wyrtki, K., 1971, Oceanographic Atlas of the International Ocean Expedition, National Science Foundation, NSF-IOE1, Washington, D.C. 